TRANSACTIONS OF THE

AMERICAN MATHEMATICAL SOCIETY

Volume 359, Number 1, January 2007, Pages 275-291

S 0002-9947(06)04009-8

Article electronically published on August 16, 2006

\title{
STRUCTURE AND STABILITY OF TRIANGLE-FREE SET SYSTEMS
}

\author{
DHRUV MUBAYI
}

\begin{abstract}
We define the notion of stability for a monotone property of set systems. This phenomenon encompasses some classical results in combinatorics, foremost among them the Erdős-Simonovits stability theorem. A triangle is a family of three sets $A, B, C$ such that $A \cap B, B \cap C, C \cap A$ are each nonempty, and $A \cap B \cap C=\emptyset$. We prove the following new theorem about the stability of triangle-free set systems.

Fix $r \geq 3$. For every $\delta>0$, there exist $\epsilon>0$ and $n_{0}=n_{0}(\epsilon, r)$ such that the following holds for all $n>n_{0}$ : if $|X|=n$ and $\mathcal{G}$ is a triangle-free family of $r$-sets of $X$ containing at least $(1-\epsilon)\left(\begin{array}{l}n-1 \\ r-1\end{array}\right)$ members, then there exists an $(n-1)$-set $S \subset X$ which contains fewer than $\delta\left(\begin{array}{l}n-1 \\ r-1\end{array}\right)$ members of $\mathcal{G}$.

This is one of the first stability theorems for a nontrivial problem in extremal set theory. Indeed, the corresponding extremal result, that for $n \geq$ $3 r / 2>4$ every triangle-free family $\mathcal{G}$ of $r$-sets of $X$ has size at most $\left(\begin{array}{l}n-1 \\ r-1\end{array}\right)$, was a longstanding conjecture of Erdös (open since 1971) that was only recently settled by Mubayi and Verstraëte (2005) for all $n \geq 3 r / 2$.
\end{abstract}

\section{INTRODUCTION}

Throughout this paper, $X$ is an $n$-element set. For any nonnegative integer $r$, we write $\left(\begin{array}{l}X \\ r\end{array}\right)$ for the family of all $r$-element subsets of $X$.

In this paper, we explicitly formulate the notion of stability for monotone properties of set systems. A Property $\mathcal{P}$ is an infinite family of set-systems closed under isomorphism. The property $\mathcal{P}$ is monotone if $\mathcal{G} \in \mathcal{P}$ and $\mathcal{G}^{\prime} \subset \mathcal{G}$ imply that $\mathcal{G}^{\prime} \in \mathcal{P}$. One can characterize monotone properties by properties not containing forbidden subsystems. More precisely, if $\mathcal{F}$ is a family of set-systems, and $\mathcal{P}_{\mathcal{F}}$ is the infinite class of set-systems containing no (isomorphic copies of a) set-system from $\mathcal{F}$, then $\mathcal{P}_{\mathcal{F}}$ is a monotone property. On the other hand, given any monotone property $\mathcal{P}$, define $\mathcal{F}$ as the collection of set-systems not contained in any member of $\mathcal{P}$. Then clearly $\mathcal{P} \subset \mathcal{P}_{\mathcal{F}}$, and the other inclusion also holds, since if $\mathcal{G} \in \mathcal{P}_{\mathcal{F}} \backslash \mathcal{P}$, then $\mathcal{G}$ is not contained in any member of $\mathcal{P}$. The definition of $\mathcal{F}$ implies that $\mathcal{G} \in \mathcal{F}$, which contradicts the fact that $\mathcal{G} \in \mathcal{P}_{\mathcal{F}}$. In the case that $\mathcal{P}$ consists of $r$-uniform set systems, we write $\mathcal{P}^{r}$.

Received by the editors March 16, 2004 and, in revised form, November 3, 2004.

2000 Mathematics Subject Classification. Primary 05C35, 05C65, 05 D05.

Key words and phrases. Extremal set theory, intersecting family, stability theorems.

The author was supported in part by NSF Grant DMS \#0400812 and by an Alfred P. Sloan Research Fellowship.

CC2006 by the author 
The property $\mathcal{P}_{n}\left(\mathcal{P}_{n}^{r}\right)$ is the subfamily of $\mathcal{P}\left(\mathcal{P}^{r}\right)$ consisting of those set systems on $n$ elements. The classical extremal problem in this regard is to determine

$$
\operatorname{ex}(n, \mathcal{F})=\max \left\{|\mathcal{G}|: \mathcal{G} \in \mathcal{P}_{n}\right\} \quad \text { or } \quad \operatorname{ex}_{r}(n, \mathcal{F})=\max \left\{|\mathcal{G}|: \mathcal{G} \in \mathcal{P}_{n}^{r}\right\},
$$

where $\mathcal{F}$ is a forbidden family for a monotone property $\mathcal{P}$. In most cases, there are only a few extremal structures $\mathcal{G}$ that achieve this maximum. For example, one of the first results of this type is Mantel's theorem from 1907 (a precursor to Turán's theorem), which states that the maximum number of edges in an $n$ vertex graph containing no triangle is $\left\lfloor n^{2} / 4\right\rfloor$, and equality holds only for $K_{\lfloor n / 2\rfloor,\lceil n / / 2\rceil}$. Perhaps the historical second to Mantel theorem is Sperner's 1928 theorem, that if $\mathcal{G} \in 2^{[n]}$ contains no two sets $A \subset B$, then $|\mathcal{G}| \leq\left(\begin{array}{c}n \\ \lfloor n / 2\rfloor\end{array}\right)$, with equality only for $\left(\begin{array}{c}{[n]} \\ \lfloor n / 2\rfloor\end{array}\right)$ or $\left(\begin{array}{c}{[n]} \\ \lceil n / 2\rceil\end{array}\right)$. The third theorem we mention in some sense forms the starting point of our investigation. The transversal number $\tau(\mathcal{A})$ of the set system $\mathcal{A}$ is the minimum size of a set that has nonempty intersection with every member of $\mathcal{A}$. Define a star to be any set system $\mathcal{A}$ with $\tau(\mathcal{A})=1$.

Theorem 1.1 (Erdös-Ko-Rado [5]). Let $n \geq 2 r$ and let $\mathcal{G} \subset\left(\begin{array}{l}X \\ r\end{array}\right)$ be an intersecting family. Then $|\mathcal{G}| \leq\left(\begin{array}{l}n-1 \\ r-1\end{array}\right)$. If $n>2 r$ and equality holds, then $\mathcal{G}$ is a star.

Upon obtaining the extremal result, and (hopefully) the extremal structures, one can go a step further, and ask whether the families in $\mathcal{P}$ of size close to $\operatorname{ex}(n, \mathcal{F})$ have structure close to the structure of one of the extremal examples (although sometimes the order of these discoveries is reversed; see the discussion that follows). Another way of viewing this picture is that we are asking for the "continuity" of the discrete $\mathcal{F}$-free structures whose size is close to the maximum. The seminal result in this regard is the Simonovits stability theorem, proved independently by Erdös and Simonovits. The Turán graph $T_{l}(n)$ is the complete $l$-partite graph on $n$ vertices with part sizes as equal as possible.

Theorem 1.2 (Erdös, Simonovits [21]). Fix $l \geq 2$. For every $\delta>0$, there exists $\epsilon>0$ and $n_{0}=n_{0}(\epsilon)$ such that the following holds for every $n>n_{0}$ : if $G$ is an $n$ vertex graph containing no copy of $K_{l+1}$ with $|G|>(1-\epsilon)\left|T_{l}(n)\right|$, then $G$ can be transformed to $T_{l}(n)$ by adding and deleting at most $\delta n^{2}$ edges.

Thus while Turán's theorem determines the structure of the extremal $K_{l+1}$-free graph, Theorem 1.2 proves the continuity of the structure of $K_{l+1}$-free graphs whose size is close to $\left|T_{l}(n)\right|$. Put plainly, a stability result usually tells us more about the problem under study than just the extremal result. However, apart from this intrinsic value, it also yields unexpected new consequences. We mention two such examples.

A basic result of Erdős-Kleitman-Rothschild 6 determines the number of labelled graphs on $n$ vertices containing no copy of $K_{l+1}$. We denote the set of these graphs by $\operatorname{Forb}(n, l+1)$. Since any subgraph of $T_{l}(n)$ is in $\operatorname{Forb}(n, l+1)$, we immediately obtain $|\operatorname{Forb}(n, l+1)| \geq 2^{\left|T_{l}(n)\right|}$. The result of Erdős-Kleitman-Rothschild states that this is sharp asymptotically in the exponent, namely, that

$$
|\operatorname{Forb}(n, l+1)|=2^{(1+o(1))\left|T_{l}(n)\right|} .
$$

The question of whether the $o(1)\left|T_{l}(n)\right|=o\left(n^{2}\right)$ term could be replaced by a term of the form $O\left(n^{2-\gamma}\right)$ for some positive $\gamma$ was open since 1976. Recently, a striking theorem of Balogh-Bollobás-Simonovits [1] proves this assertion (for a much 
larger class of forbidden subgraphs), and one of the main tools in the proof is a strengthening of Theorem 1.2 .

Another approach first used by Simonovits 21] for graphs, and more recently by several authors 11, 13, 14, 16, 19, for hypergraphs, is to prove an exact result using a stability result. More precisely, one can determine $\operatorname{ex}_{r}(n, \mathcal{F})$ exactly by first determining the asymptotic value, then using a stability theorem to prove that any structure with the extremal number of sets is the unique extremal structure. This is especially valuable in extremal hypergraph theory, where exact results are rare, and any new approach gives insight to the governing phenomenon of the problems.

Here we provide a common formulation for these and other problems. In what follows, we write $\mathcal{G}_{n}$ for a set system whose underlying set has size $n$. The formulation below applies as well to $\mathcal{P}^{r}$ even though we write it only for $\mathcal{P}$. Recall that the forbidden family for $\mathcal{P}$ is the collection $\mathcal{F}$ of set-systems not contained in any member of $\mathcal{P}$.

Definition. Let $t>0$ be an integer, let $\mathcal{P}$ be a monotone property of set systems, and let $\mathcal{F}$ be a forbidden family for $\mathcal{P}$. The property $\mathcal{P}$ is $t$-stable if there exists $m_{0}=m_{0}(\mathcal{F})$ and set systems $\mathcal{H}_{m}^{1}, \ldots, \mathcal{H}_{m}^{t}$ for every $m>m_{0}$ such that the following holds: for every $\delta>0$, there exists $\epsilon>0$ and $n_{0}=n_{0}(\epsilon)$ such that for all $n>n_{0}$, if $\mathcal{G}_{n} \in \mathcal{P}_{n}$ with

$$
\left|\mathcal{G}_{n}\right|>(1-\epsilon) \operatorname{ex}(n, \mathcal{F})
$$

then $\mathcal{G}_{n}$ can be transformed to some $\mathcal{H}_{n}^{i}$ by adding and removing at most $\delta\left|\mathcal{G}_{n}\right|$ sets. Say that $\mathcal{P}$ is stable if it is 1-stable.

Our interest is in developing stability results in extremal set theory. Perhaps the first such result is the theorem of Hilton and Milner 12, which determines the maximum size of an intersecting family $\mathcal{G} \subset\left(\begin{array}{c}{[n]} \\ r\end{array}\right)$ with $\tau(\mathcal{G})>1$. An easy consequence of their result is that if $r$ is fixed, $n>n_{0}(r)$, and $|G|>c n^{r-2}$ for some constant $c$ depending only on $r$, then $\tau(G)=1$ (it is likely that this was also known to Erdös-Ko-Rado). We prove a stability result for set systems where the forbidden configuration is a generalization of that in Mantel's theorem.

A triangle is a family of three sets $A, B, C$ such that $A \cap B, B \cap C, C \cap A$ are each nonempty, and $A \cap B \cap C=\emptyset$. Let $f(r, n)$ denote the maximum size of a family $\mathcal{A} \subset\left(\begin{array}{l}X \\ r\end{array}\right)$ containing no triangle. Mantel's theorem states that $f(2, n)=\left\lfloor n^{2} / 4\right\rfloor$, and this motivated Erdös [4] to ask for the determination of $f(r, n)$ for $r>2$. He conjectured that

$$
f(r, n)=\left(\begin{array}{l}
n-1 \\
r-1
\end{array}\right) \quad \text { for } \quad n \geq 3 r / 2 .
$$

(Actually, in 44 it is stated more as a question, and $n \geq 3 r / 2$ is not explicitly mentioned, but later, e.g., in [3, 8, (1.1) is referred to as a conjecture of Erdös.)

This conjecture attracted quite a few researchers, including Chvátal, Bermond, Frankl and Füredi [3, 2, 7, 8, 9, whose work resulted in its solution for $3 r / 2 \leq$ $n \leq 2 r$, and $n>n_{0}(r)$, where $n_{0}(r)$ is an unspecified but exponentially growing function of $r$. Recently, the author and Verstraëte [18] proved (1.1) for all $n \geq 3 r / 2$ using a new combinatorial method. We take this one step further and prove that the property of being triangle-free is stable, with the unique extremal configuration being a star with the maximum number of sets. The following is an equivalent formulation, and is the main result of this paper. 
Theorem 1.3 (Main Result). Fix $r \geq 3$. For every $\delta>0$, there exist $\epsilon>0$ and $n_{0}=n_{0}(\epsilon, r)$ such that the following holds for all $n>n_{0}$ : if $|X|=n$ and $\mathcal{G} \subset\left(\begin{array}{l}X \\ r\end{array}\right)$ contains no triangle, $|\mathcal{G}|>(1-\epsilon)\left(\begin{array}{l}n-1 \\ r-1\end{array}\right)$, then there exists an $S \subset X$ with $|S|=n-1$ such that $\left|\mathcal{G} \cap\left(\begin{array}{c}S \\ r\end{array}\right)\right| \leq \delta\left(\begin{array}{c}n-1 \\ r-1\end{array}\right)$.

Chvátal 3 proved (1.1) for $r=3$. In fact, he proved the more general statement that if $n \geq r+2 \geq 5, \mathcal{A} \subset\left(\begin{array}{l}X \\ r\end{array}\right)$, and $|\mathcal{A}|>\left(\begin{array}{l}n-1 \\ r-1\end{array}\right)$, then $\mathcal{A}$ contains $r$ sets $A_{1}, \ldots, A_{r}$ such that every $r-1$ of them have nonempty intersection, but $\bigcap_{i} A_{i}=\emptyset$. This configuration is also called an $(r-1)$-dimensional simplex. Chvátal generalized (1.1) as follows.

Conjecture 1.4 (Chvátal). Let $r \geq d+1 \geq 3, n \geq r(d+1) / d$ and $\mathcal{A} \subset\left(\begin{array}{l}X \\ r\end{array}\right)$. If $\mathcal{A}$ contains no d-dimensional simplex, then $|\mathcal{A}| \leq\left(\begin{array}{l}n-1 \\ r-1\end{array}\right)$. If equality holds, then $\mathcal{A}$ is a star.

Frankl and Füredi 9] proved Conjecture 1.4 for $n>n_{0}(r)$, where $n_{0}(r)$ is exponential in $r$. It would be very surprising if the property of containing no $d$ dimensional simplex was not stable. Nevertheless, we lack the tools to settle the following conjecture at present.

Conjecture 1.5. Fix $r \geq d+1 \geq 3$. For every $\delta>0$, there exist $\epsilon>0$ and $n_{0}=n_{0}(\epsilon, r)$ such that the following holds for all $n>n_{0}$ : if $|X|=n$ and $\mathcal{G} \subset\left(\begin{array}{l}X \\ r\end{array}\right)$ contains no d-dimensional simplex and $|\mathcal{G}|>(1-\epsilon)\left(\begin{array}{l}n-1 \\ r-1\end{array}\right)$, then there exists an $S \subset X$ with $|S|=n-1$ such that $\left|\mathcal{G} \cap\left(\begin{array}{l}S \\ r\end{array}\right)\right| \leq \delta\left(\begin{array}{l}n-1 \\ r-1\end{array}\right)$.

In Section 2 we provide additional notation for set systems. The main combinatorial lemmas are proved in Section 3, where a bipartite version of Erdős' question is considered. Section 4 contains two technical lemmas, and the proof of Theorem 1.3 is completed in Section 5. In Section 6 we list a host of open problems, where stability results could be obtained.

\section{Notation}

For $\mathcal{A} \subset\left(\begin{array}{l}X \\ r\end{array}\right)$, let $V(\mathcal{A})=\bigcup_{A \in \mathcal{A}} A$ and $n(\mathcal{A})=|V(\mathcal{A})|$. For $Y \subset X$, we define $\mathcal{A}-Y=\mathcal{A} \cap\left(\begin{array}{c}X \backslash Y \\ r\end{array}\right)$. When $Y=\{y\}$, we write $\mathcal{A}-y$ instead of $\mathcal{A}-\{y\}$.

The following six definitions and associated notations will be used repeatedly throughout the paper.

Sum of families. The sum of families $\mathcal{A}_{1}, \mathcal{A}_{2}, \ldots, \mathcal{A}_{t}$, denoted $\sum_{i} \mathcal{A}_{i}$, is the family of all sets in each $\mathcal{A}_{i}$. Note that $\sum \mathcal{A}_{i}$ may have repeated sets, even if none of the $\mathcal{A}_{i}$ have repeated sets.

Trace of a set. The trace of a set $Y$ in $\mathcal{A}$ is defined by $\operatorname{tr}(Y)=\operatorname{tr}_{\mathcal{A}}(Y)=\{A \subset$ $X \backslash Y: A \cup Y \in \mathcal{A}\}$. We define $\operatorname{tr}(\mathcal{A})=\sum_{x \in X} \operatorname{tr}(x)$.

Degree. The degree of a vertex $x$ in $\mathcal{A}$ is $\operatorname{deg}(x)=\operatorname{deg}_{\mathcal{A}}(x)=\left|\operatorname{tr}_{\mathcal{A}}(x)\right|$.

The families $\mathcal{S}_{x}$ and $\mathcal{L}_{x}$. Let $\mathcal{A}$ be an $r$-uniform family of sets in $X$ and $x \in X$. Then we define

$$
\mathcal{S}_{x}=\{Y \in \operatorname{tr}(x):|\operatorname{tr}(Y)|=1\} \quad \text { and } \quad \mathcal{L}_{x}=\operatorname{tr}(x) \backslash \mathcal{S}_{x} .
$$

We write $\mathcal{S}=\sum_{x \in X} \mathcal{S}_{x}$ and $\mathcal{L}=\sum_{x \in X} \mathcal{L}_{x}=\operatorname{tr}(\mathcal{A}) \backslash \mathcal{S}$. Note that if $A \in \mathcal{L}_{x}$, then there exists $y \neq x$ such that $A \in \mathcal{L}_{y}$. 
Paths and connectivity. A path in $\mathcal{A}$ is a family $\mathcal{P}$ of sets $A_{1}, A_{2}, \ldots$ such that $A_{i} \cap A_{j} \neq \emptyset$ if and only if $|i-j| \leq 1$. Family $\mathcal{A}$ is connected if every pair of vertices in $V(\mathcal{A})$ is contained in some path in $\mathcal{A}$. A component of $\mathcal{A}$ is a maximal nonempty connected subfamily of $\mathcal{A}$.

Shadow. The shadow $\partial \mathcal{G}$ of a set system $\mathcal{G} \subset\left(\begin{array}{l}X \\ r\end{array}\right)$ is defined by

$$
\partial \mathcal{G}=\left\{S \in\left(\begin{array}{c}
X \\
r-1
\end{array}\right): \text { there exists } T \in \mathcal{G} \text { with } S \subset T\right\} .
$$

Throughout the paper, the Greek letters $\epsilon, \delta$, etc. are real numbers, and $m, n, r$, $s, t$, etc. are integers.

\section{Combinatorial LEMmas AND A BIPARTite FORMUlation}

The proof techniques of this paper rely on carefully counting various subsets in a set system. Many of the crucial ideas that will be used repeatedly are presented in this section.

Lemma 3.1. Let $n>r \geq 3$, let $\mathcal{G} \in\left(\begin{array}{l}X \\ r\end{array}\right)$ be triangle-free, and $z \in X$. Suppose that $\mathcal{K}_{1}, \ldots, \mathcal{K}_{s}$ are the components of $\mathcal{L}_{z}$. Let $\mathcal{K}_{i}^{\prime}$ consist of $\mathcal{K}_{i}$ together with all the sets in $\mathcal{S}_{z}$ that contain a point in $V\left(\mathcal{K}_{i}\right)$. Then $\mathcal{K}_{i}^{\prime}$ is an intersecting family.

Proof. Suppose, for a contradiction, that $\mathcal{K}_{i}^{\prime}$ contains disjoint sets $A, B$. Since $\mathcal{K}_{i}$ is connected, $\mathcal{K}_{i} \cup\{A, B\}$ is also connected. Let $\mathcal{P}$ be a shortest $A, B$-path in $\mathcal{K}_{i} \cup\{A, B\}$, and let $C$ be the set on $\mathcal{P}$ immediately following $A$. Since $C \not \subset\{A, B\}$, we know that $C \in \mathcal{K}_{i}$. Let $D$ be the set on $\mathcal{P}$ immediately following $C$ (possibly $D=B$ ). Because $\mathcal{P}$ is a shortest path, $A \cap D=\emptyset$. Since $C \in \mathcal{K}_{i} \subset \mathcal{L}_{z}$, there exists $z^{\prime} \neq z$ with $C \in \mathcal{L}_{z^{\prime}}$. Consequently, the sets

$$
A \cup z, \quad C \cup z^{\prime}, \quad D \cup z
$$

form a triangle in $\mathcal{G}$. This contradiction implies that $\mathcal{K}_{i}^{\prime}$ is an intersecting family.

Lemma 3.2. Let $n>r \geq 3$, and $\mathcal{G} \in\left(\begin{array}{c}X \\ r\end{array}\right)$ be triangle-free. Then every $z \in X$ satisfies

$$
\left|\mathcal{L}_{z}\right| \leq\left(\begin{array}{l}
n-1 \\
r-2
\end{array}\right)
$$

Proof. Let $\mathcal{K}_{1}, \mathcal{K}_{1}, \ldots, \mathcal{K}_{s}$ be the components of $\mathcal{L}_{z}$. Lemma 3.1 implies that each $\mathcal{K}_{i} \subset\left(\begin{array}{c}X \\ r-1\end{array}\right)$ is intersecting. Let $n_{i}=n\left(\mathcal{K}_{i}\right)$. If $n_{i} \geq 2(r-1)$, then Theorem 1.1 yields $\left|\mathcal{K}_{i}\right| \leq\left(\begin{array}{c}n_{i}-1 \\ r-2\end{array}\right) \leq\left(\begin{array}{c}n_{i} \\ r-2\end{array}\right)$. If $n_{i} \leq 2 r-3$, then $\left|\mathcal{K}_{i}^{\prime}\right| \leq\left(\begin{array}{c}n_{i} \\ r-1\end{array}\right)$, and again this is at most $\left(\begin{array}{c}n_{i} \\ r-2\end{array}\right)$. Therefore, convexity of binomial coefficients yields

$$
\left|\mathcal{L}_{z}\right|=\sum_{i=1}^{s}\left|\mathcal{K}_{i}\right| \leq \sum_{i=1}^{s}\left(\begin{array}{c}
n_{i} \\
r-2
\end{array}\right) \leq\left(\begin{array}{c}
n-1 \\
r-2
\end{array}\right)
$$

where the last inequality holds since $\sum_{i} n_{i} \leq n-1$.

The crux of our method in this paper is that we relate $\sum_{z}\left|\mathcal{L}_{z}\right|$ to $|\mathcal{G}|$ by counting the contribution to the sum from the $(r-1)$-sets in $\partial \mathcal{G}$. This is illustrated in Proposition 3.3 below, which can be viewed as a bipartite version of the extremal problem for triangle-free families. This is one of the crucial new steps needed to prove Theorem 1.3, and a variant of the counting argument in its proof will be used again later. 
Proposition 3.3. Let $n>r \geq 3$ and $0<\epsilon<1 / 10$. Suppose that $X$ has partition $A \cup B$ with $|B| \leq \epsilon n$. If $\mathcal{G} \subset\left(\begin{array}{l}X \\ r\end{array}\right)$ is triangle-free, and every $E \in \mathcal{G}$ satisfies $|E \cap B| \geq 2$, then $|\mathcal{G}|<r \epsilon\left(\begin{array}{c}n \\ r-1\end{array}\right)$.

Proof. Since each set in $\mathcal{G}$ contains at least two elements in $B$,

$$
2|\mathcal{G}| \leq \sum_{x \in B} \operatorname{deg}(x)=\sum_{x \in B}\left(\left|\mathcal{S}_{x}\right|+\left|\mathcal{L}_{x}\right|\right)=\sum_{x \in B}\left|\mathcal{S}_{x}\right|+\sum_{x \in B}\left|\mathcal{L}_{x}\right|
$$

The condition of the theorem also implies that if $E^{\prime} \in \partial \mathcal{G}$, then $\left|E^{\prime} \cap B\right| \geq 1$. Since $\mathcal{S}_{B}=\sum_{x \in B} \mathcal{S}_{x} \subset \partial \mathcal{G}$, the same conclusion holds for sets in $\mathcal{S}_{B}$. For every $E \in \mathcal{S}_{B}$, there is exactly one $x$ for which $E \in \mathcal{S}_{x}$. Therefore

$$
\sum_{x \in B}\left|\mathcal{S}_{x}\right|=\left|\mathcal{S}_{B}\right| \leq|B|\left(\begin{array}{c}
n-1 \\
r-2
\end{array}\right) \leq \epsilon n\left(\begin{array}{c}
n-1 \\
r-2
\end{array}\right) .
$$

Since $\mathcal{G}$ is triangle-free, Lemma 3.2 implies that $\left|\mathcal{L}_{x}\right| \leq\left(\begin{array}{l}n-1 \\ r-2\end{array}\right)$ for every $x \in B$ which yields

$$
\sum_{x \in B}\left|\mathcal{L}_{x}\right|=\left|\mathcal{L}_{B}\right| \leq|B|\left(\begin{array}{c}
n-1 \\
r-2
\end{array}\right) \leq \epsilon n\left(\begin{array}{c}
n-1 \\
r-2
\end{array}\right) .
$$

Now (3.3), (3.4) and (3.5) yield

$$
2|\mathcal{G}| \leq 2 \epsilon n\left(\begin{array}{l}
n-1 \\
r-2
\end{array}\right)<2 r \epsilon\left(\begin{array}{c}
n \\
r-1
\end{array}\right) .
$$

This gives the required bound on $|\mathcal{G}|$.

\section{TWO MORE LEMMAS}

The following lemma follows from a result of Hilton and Milner [12. We present our own proof for the sake of completeness. It includes a proof of the Erdös-KoRado Theorem (Theorem 1.1) for large $n$.

Lemma 4.1. Let $n>t \geq 3$ and $\mathcal{G} \subset\left(\begin{array}{c}X \\ t\end{array}\right)$ be an intersecting family with $|\mathcal{G}|>$ $3+\left(\begin{array}{c}3 t-3 \\ 2\end{array}\right)\left(\begin{array}{c}n-2 \\ t-2\end{array}\right)$. Then $\mathcal{G}$ is star.

Proof. Suppose first that there exists $E \in \mathcal{G}$ for which $|E \cap F| \geq 2$ for every $F \in \mathcal{G}$. Then

$$
|\mathcal{G}| \leq 1+\left(\begin{array}{l}
t \\
2
\end{array}\right)\left(\begin{array}{c}
n-2 \\
t-2
\end{array}\right)<3+\left(\begin{array}{c}
3 t-3 \\
2
\end{array}\right)\left(\begin{array}{c}
n-2 \\
t-2
\end{array}\right)
$$

a contradiction. We may therefore assume that there exists some $x \in V(\mathcal{G})$ and distinct sets $E, E^{\prime} \in \mathcal{G}$ such that $E \cap E^{\prime}=\{x\}$. Suppose that $x \notin F$ for some $F \in \mathcal{G}$. Since $\mathcal{G}$ is an intersecting family, $\left\{E, E^{\prime}, F\right\}$ forms a triangle, and therefore every set in $\mathcal{G}$ contains at least two elements from $E \cup E^{\prime} \cup F$. Consequently,

$$
|\mathcal{G}| \leq 3+\left(\begin{array}{c}
\left|E \cup E^{\prime} \cup F\right| \\
2
\end{array}\right)\left(\begin{array}{c}
n-2 \\
t-2
\end{array}\right) \leq 3+\left(\begin{array}{c}
3 t-3 \\
2
\end{array}\right)\left(\begin{array}{c}
n-2 \\
t-2
\end{array}\right)
$$

a contradiction. Hence $x \in F$ for every $F \in \mathcal{G}$, and therefore $\mathcal{G}$ is a star.

Lemma 4.2. For $t \geq 4$ and any $0<\epsilon^{\prime}<1 / 2 t$, let

$$
\epsilon \leq \frac{\epsilon^{\prime}(t-3)}{4} \quad \text { and } \quad n_{0}=\frac{t}{\epsilon}+1
$$


If $n>n_{0}$ and $n_{1} \geq n_{2} \geq \cdots \geq n_{s} \geq t$ with $\sum_{i} n_{1} \leq n-1$ and

$$
\sum_{i=1}^{s}\left(\begin{array}{c}
n_{i} \\
t-2
\end{array}\right)>(1-2 \epsilon)\left(\begin{array}{c}
n-2 \\
t-2
\end{array}\right)
$$

then $n_{1}>\left(1-\epsilon^{\prime}\right)(n-1)$.

Proof. First observe that $\epsilon^{\prime}<1$ yields

$$
\frac{n-1}{t-2}\left(\begin{array}{c}
\left(1-\epsilon^{\prime}\right)(n-2) \\
t-3
\end{array}\right)<\left(1-\epsilon^{\prime}\right)^{t-3} \frac{n-1}{n-t+1}\left(\begin{array}{c}
n-2 \\
t-2
\end{array}\right) .
$$

Now $\epsilon \leq \epsilon^{\prime}(t-3) / 4<1 / 6$ implies that

$$
\left(1-\epsilon^{\prime}\right)^{t-3}<e^{-\epsilon^{\prime}(t-3)}=e^{-4 \epsilon}<1-4 \epsilon+8 \epsilon^{2}<(1-\epsilon)(1-2 \epsilon),
$$

and the choice of $n_{0}$ implies that

$$
\frac{n-1}{n-t+1}<\frac{1}{1-\epsilon}
$$

Using these two bounds in (4.1) we obtain

$$
\frac{n-1}{t-2}\left(\begin{array}{c}
\left(1-\epsilon^{\prime}\right)(n-2) \\
t-3
\end{array}\right)<(1-2 \epsilon)\left(\begin{array}{c}
n-2 \\
t-2
\end{array}\right) .
$$

We interpret $\sum_{i=1}^{s}\left(\begin{array}{c}n_{i} \\ t-2\end{array}\right)$ as the number of $(t-2)$-sets in $\mathcal{H}=\bigcup_{i=1}^{s}\left(\begin{array}{c}X_{i} \\ t-2\end{array}\right)$, where $X_{1}, \ldots, X_{s}$ are disjoint sets with $\left|X_{i}\right|=n_{i}$. Thus for every $x \in V(\mathcal{H})$, we have $\operatorname{deg}_{\mathcal{H}}(x) \leq\left(\begin{array}{c}n_{1}-1 \\ t-3\end{array}\right)$, with equality only if $x \in X_{i}$ and $n_{i}=n_{1}$.

Suppose, for a contradiction, that $n_{1} \leq\left(1-\epsilon^{\prime}\right)(n-1)$. Then, since $n(\mathcal{H})=$ $\sum_{i} n_{i} \leq n-1$

$$
\sum_{i=1}^{s}\left(\begin{array}{c}
n_{i} \\
t-2
\end{array}\right)=|\mathcal{H}|=\frac{\sum_{x \in V(\mathcal{H})} \operatorname{deg}(x)}{t-2}<\frac{n-1}{t-2}\left(\begin{array}{c}
\left(1-\epsilon^{\prime}\right)(n-2) \\
t-3
\end{array}\right) .
$$

By (4.2), this is at most $(1-2 \epsilon)\left(\begin{array}{c}n-2 \\ t-2\end{array}\right)$, which is a contradiction.

\section{Proof of Theorem 1.3}

In this section we complete the proof of the Main Result.

Proof of Theorem 1.3. Fix $r \geq 3$ and $\delta$ as in the theorem. When $r \geq 4$, let

$$
\epsilon^{\prime \prime} \leq \frac{\delta}{5 r}, \quad \epsilon^{\prime} \leq \frac{\epsilon^{\prime \prime}}{4}, \quad \epsilon \leq \frac{\epsilon^{\prime}(r-3)}{4}, \quad n_{0}=\max \left\{\frac{r}{\epsilon}+1,5 r^{3}\right\} .
$$

When $r=3$, let

$$
\epsilon^{\prime} \leq \frac{\delta}{6}, \quad \epsilon \leq\left(\frac{\epsilon^{\prime}}{114}\right)^{2}, \quad n_{0}=\frac{4}{\epsilon}+1
$$

In both cases, we can think of the flow of constants as

$$
0<\frac{1}{n_{0}} \ll \epsilon \ll \epsilon^{\prime} \ll \epsilon^{\prime \prime} \ll \delta,
$$

although in the case $r \geq 8$, it is possible for $\epsilon>\epsilon^{\prime}$. In particular, for all $r \geq 3$, we can let

$$
\epsilon=\max \left\{\left(\frac{\delta}{684}\right)^{2}, \frac{\delta(r-3)}{80 r}\right\} .
$$


Let $\mathcal{G} \subset\left(\begin{array}{l}X \\ r\end{array}\right)$ be a triangle-free family with $|\mathcal{G}|>(1-\epsilon)\left(\begin{array}{l}n-1 \\ r-1\end{array}\right)$. Our strategy is to obtain the $(n-1)$-set $S$ in the conclusion of the theorem in three steps:

1) Find a vertex $w$ with $\left|\mathcal{L}_{w}\right|$ very large.

2) Study the structure of $\left|\mathcal{L}_{w}\right|$, in particular, show that it contains a large star with center $x$. In the case $r \geq 4$, this follows from Lemma 4.1. However, when $r=3,\left|\mathcal{L}_{w}\right|$ can possibly consist of disjoint (graph) triangles. Consequently, the proof of this step for $r=3$ is more lengthy and difficult.

3) Define $S=X \backslash\{x\}$ and show that $S$ satisfies the requirements of the theorem, since otherwise we obtain a triangle in $\mathcal{G}$ (this is the most lengthy and difficult part of the proof for $r \geq 4$ ).

Step 1. We begin with the following equation which is an easy double counting exercise:

$$
r|\mathcal{G}|=\sum_{x \in X} \operatorname{deg}(x)=\sum_{x \in X}\left(\left|\mathcal{S}_{x}\right|+\left|\mathcal{L}_{x}\right|\right)=\sum_{x \in X}\left|\mathcal{S}_{x}\right|+\sum_{x \in X}\left|\mathcal{L}_{x}\right| .
$$

Since $\sum_{x}\left|\mathcal{S}_{x}\right|=|\mathcal{S}| \leq\left(\begin{array}{c}n \\ r-1\end{array}\right)$, there exists $w \in X$ for which

$$
\begin{aligned}
\left|\mathcal{L}_{w}\right| \geq \frac{r|\mathcal{G}|-\left(\begin{array}{c}
n \\
r-1
\end{array}\right)}{n} & >\frac{r(1-\epsilon)\left(\begin{array}{c}
n-1 \\
r-1
\end{array}\right)-\left(\begin{array}{c}
n \\
r-1
\end{array}\right)}{n} \\
& >(1-2 \epsilon)\left(\begin{array}{l}
n-2 \\
r-2
\end{array}\right),
\end{aligned}
$$

where the last inequality follows from a short calculation and the fact that $n>n_{0}$.

This concludes Step 1. We now separate the cases $r=3$ and $r \geq 4$, since the arguments are quite different.

Step $2(r \geq 4)$. As in Lemma 3.2. let $\mathcal{K}_{1}, \mathcal{K}_{1}, \ldots, \mathcal{K}_{s}$ be the components of $\mathcal{L}_{w}$. Then by Lemma 3.1 each $\mathcal{K}_{i}$ is an intersecting family, and (5.1) and (3.2) give

$$
(1-2 \epsilon)\left(\begin{array}{c}
n-2 \\
r-2
\end{array}\right)<\left|\mathcal{L}_{w}\right|=\sum_{i=1}^{s}\left|\mathcal{K}_{i}\right| \leq \sum_{i=1}^{s}\left(\begin{array}{c}
n_{i} \\
r-2
\end{array}\right)
$$

where $\sum_{i} n_{i} \leq n-1$.

We may now apply Lemma 4.2 with $t=r$ to (5.2) and conclude that

$$
n_{1} \geq\left(1-\epsilon^{\prime}\right)(n-1) \text {. }
$$

Moreover, convexity of binomial coefficients yields

$\left|\mathcal{L}_{w}\right|=\left|\mathcal{K}_{1}\right|+\sum_{i=2}^{s}\left|\mathcal{K}_{i}\right| \leq\left|\mathcal{K}_{1}\right|+\sum_{i=2}^{s}\left(\begin{array}{c}n_{i} \\ r-2\end{array}\right)<\left|\mathcal{K}_{1}\right|+\left(\begin{array}{c}n-n_{1} \\ r-2\end{array}\right)<\left|\mathcal{K}_{1}\right|+\epsilon^{\prime}\left(\begin{array}{c}n-2 \\ r-2\end{array}\right)$.

Since $\left|\mathcal{L}_{w}\right|>(1-2 \epsilon)\left(\begin{array}{l}n-2 \\ r-2\end{array}\right)$, we have by (5.4) and $n>n_{0} \geq 5 r^{3}$,

$$
\begin{aligned}
\left|\mathcal{K}_{1}\right| & >\left(1-2 \epsilon-\epsilon^{\prime}\right)\left(\begin{array}{l}
n-2 \\
r-2
\end{array}\right) \\
& >\left(1-2 \epsilon^{\prime}\right)\left(\begin{array}{c}
n-2 \\
r-2
\end{array}\right) \\
& >3+\left(\begin{array}{c}
3 r-6 \\
2
\end{array}\right)\left(\begin{array}{l}
n-2 \\
r-3
\end{array}\right) .
\end{aligned}
$$


Since $\mathcal{K}_{1}$ is an intersecting family of $(r-1)$-sets, (5.6) and Lemma 4.1 with $t=r-1$ imply that $\mathcal{K}_{1}$ is a star. Let $x$ be the center of $\mathcal{K}_{1}$. This concludes Step 2.

Step $3(r \geq 4)$. The rest of the proof is devoted to proving that $\mathcal{G}-x=\mathcal{G} \cap(\underset{r}{X \backslash\{x\}})$ satisfies

$$
|\mathcal{G}-x| \leq \delta\left(\begin{array}{l}
n-1 \\
r-1
\end{array}\right)
$$

Let

$$
\mathcal{G}_{w, x}=\operatorname{tr}_{\mathcal{G}}(\{w, x\})=\left\{E \in\left(\begin{array}{c}
X \\
r-2
\end{array}\right): E \cup\{w, x\} \in \mathcal{G}\right\} .
$$

Claim 1. There are disjoint $(r-3)$-sets $S_{1}, S_{2} \subset V\left(\mathcal{K}_{1}\right)$ such that for each $i \in$ $\{1,2\}$,

$$
\left|\left\{y \in V\left(\mathcal{K}_{1}\right): S_{i} \cup\{w, x, y\} \in \mathcal{G}\right\}\right| \geq\left(1-\epsilon^{\prime \prime}\right)(n-r+1) .
$$

Proof. Let $t$ be the number of $(r-3)$-sets $T \subset V\left(\mathcal{K}_{1}\right) \backslash\{x\}$ satisfying

$$
\operatorname{tr}_{\mathcal{G}_{w, x}}(T)=\left|\left\{y \in V\left(\mathcal{K}_{1}\right): T \cup\{w, x, y\} \in \mathcal{G}\right\}\right|>\left(1-\epsilon^{\prime \prime}\right)(n-r+1) .
$$

Then

$$
\begin{aligned}
(r-2)\left|\mathcal{K}_{1}\right|=\left(\begin{array}{l}
r-2 \\
r-3
\end{array}\right)\left|\mathcal{K}_{1}\right| & =\sum_{\substack{T^{\prime} \subset V\left(\mathcal{K}_{1}\right) \backslash\{x\} \\
\left|T^{\prime}\right|=r-3}} \operatorname{tr}_{\mathcal{G}_{w, x}}\left(T^{\prime}\right) \\
& \leq t(n-r+1)+\left[\left(\begin{array}{l}
n-2 \\
r-3
\end{array}\right)-t\right]\left(1-\epsilon^{\prime \prime}\right)(n-r+1) .
\end{aligned}
$$

This implies that

$$
t>\frac{(r-2)\left|\mathcal{K}_{1}\right|-\left(\begin{array}{c}
n-2 \\
r-3
\end{array}\right)\left(1-\epsilon^{\prime \prime}\right)(n-r+1)}{\epsilon^{\prime \prime}(n-r+1)} .
$$

By (5.5), this is at least

$$
\begin{aligned}
& \frac{(r-2)\left(1-2 \epsilon^{\prime}\right)\left(\begin{array}{l}
n-2 \\
r-2
\end{array}\right)-\left(\begin{array}{l}
n-2 \\
r-3
\end{array}\right)\left(1-\epsilon^{\prime \prime}\right)(n-r+1)}{\epsilon^{\prime \prime}(n-r+1)} \\
= & \frac{1-2 \epsilon^{\prime}}{\epsilon^{\prime \prime}}\left(\begin{array}{l}
n-2 \\
r-3
\end{array}\right)-\frac{1-\epsilon^{\prime \prime}}{\epsilon^{\prime \prime}}\left(\begin{array}{l}
n-2 \\
r-3
\end{array}\right) \\
= & \frac{\epsilon^{\prime \prime}-2 \epsilon^{\prime}}{\epsilon^{\prime \prime}}\left(\begin{array}{l}
n-2 \\
r-3
\end{array}\right) \\
\geq & \frac{1}{2}\left(\begin{array}{l}
n-2 \\
r-3
\end{array}\right)>\left(\begin{array}{l}
n-3 \\
r-4
\end{array}\right),
\end{aligned}
$$

where the second-to-the-last inequality holds since $\epsilon^{\prime} \leq \epsilon^{\prime \prime} / 4$ and the last inequality holds since $n>2 r$. Thus the Erdős-Ko-Rado theorem (Theorem 1.1) applies to give the sets $S_{1}$ and $S_{2}$.

Define, for $i \in\{1,2\}$,

$$
A_{i}=\left\{y \in V\left(\mathcal{K}_{1}\right): S_{i} \cup\{w, x, y\} \in \mathcal{G}\right\},
$$

and let

$$
A=A_{1} \cap A_{2}=\left\{y \in V\left(\mathcal{K}_{1}\right): S_{i} \cup\{w, x, y\} \in \mathcal{G} \text { for } i=1,2\right\} .
$$

Set

$$
B=X \backslash(A \cup\{w, x\}) .
$$


Note that $S_{1} \cup S_{2} \subset B$. By Claim 1, $\left|A_{i}\right| \geq\left(1-\epsilon^{\prime \prime}\right)(n-r+1)$ for $i=1,2$. Therefore

$$
|A|=\left|A_{1} \cap A_{2}\right| \geq 2\left(1-\epsilon^{\prime \prime}\right)(n-r+1)-n .
$$

It now follows that

$$
|B| \leq n-|A| \leq 2 n-2\left(1-\epsilon^{\prime \prime}\right)(n-r+1) \leq 3 \epsilon^{\prime \prime} n .
$$

Partition $\mathcal{G}-x$ into $\mathcal{G}_{1} \cup \mathcal{G}_{2}$, where

$$
\mathcal{G}_{1}=\{E \in \mathcal{G}-x:|E \cap B| \leq 1\} \quad \text { and } \quad \mathcal{G}_{2}=\{E \in \mathcal{G}-x:|E \cap B| \geq 2\} .
$$

Since $\mathcal{G}_{2}$ is triangle-free, it satisfies the conditions of Proposition 3.3 with $3 \epsilon^{\prime \prime}$ playing the role of $\epsilon$, and therefore

$$
\left|\mathcal{G}_{2}\right| \leq 3 r \epsilon^{\prime \prime}\left(\begin{array}{c}
n \\
r-1
\end{array}\right) .
$$

We now focus on $\mathcal{G}_{1}$. Note that

$$
\mathcal{G}_{1}-w=\left\{E \in \mathcal{G}_{1}: w \notin E\right\}=\{E \in \mathcal{G}-\{w, x\}:|E \cap B| \leq 1\} .
$$

Claim 2. $\mathcal{G}_{1}-w=\emptyset$.

Proof. Suppose, for a contradiction, that $E \in \mathcal{G}_{1}-w$. Since $|E \cap B| \leq 1$ and $S_{1} \cup S_{2} \subset B$, we have $\left|E \cap\left(S_{1} \cup S_{2}\right)\right| \leq 1$. Furthermore, by Claim 1 we know that $S_{1}$ and $S_{2}$ are disjoint. Therefore $E$ has an empty intersection with at least one $S_{i}$, say $S_{1}$. Since at least $r-1$ elements of $E$ lie in $A$ and since $r \geq 3$, there exist distinct elements $y, y^{\prime} \in A \cap E$. By the definition of $A$, we know that

$$
S_{1} \cup\{w, x, y\} \in \mathcal{G} \quad \text { and } \quad S_{1} \cup\left\{w, x, y^{\prime}\right\} \in \mathcal{G} .
$$

Since $E \cap\left(S_{1} \cup\{w, x\}\right)=\emptyset$, these two sets together with $E$ form a triangle in $\mathcal{G}$, which is a contradiction.

Note that Claim 2 says that all sets of $\mathcal{G}_{1}$ contain $w$. This implies that $\left|\mathcal{G}_{1}\right|=$ $\left|\operatorname{tr}_{\mathcal{G}_{1}}(w)\right|$. Since $\mathcal{G}_{1} \subset \mathcal{G}-\{x\}$, we have $\operatorname{tr}_{\mathcal{G}_{1}}(w) \subset \operatorname{tr}_{\mathcal{G}-x}(w)$. Therefore

$$
\left|\mathcal{G}_{1}\right| \leq\left|\operatorname{tr}_{\mathcal{G}-x}(w)\right| \text {. }
$$

Our final step is to bound $\left|\operatorname{tr}_{\mathcal{G}-x}(w)\right|$.

Partition $\operatorname{tr}_{\mathcal{G}-x}(w)$ into $\operatorname{tr}_{\mathcal{G}-x}^{0}(w) \cup \operatorname{tr}_{\mathcal{G}-x}^{1}(w)$, where

$$
\begin{gathered}
\operatorname{tr}_{\mathcal{G}-x}^{0}(w)=\left\{E \in \operatorname{tr}_{\mathcal{G}-x}(w):|E \cap B|=0\right\} \text { and } \\
\operatorname{tr}_{\mathcal{G}-x}^{1}(w)=\left\{E \in \operatorname{tr}_{\mathcal{G}-x}(w):|E \cap B| \geq 1\right\} .
\end{gathered}
$$

Consider $E \in \operatorname{tr}_{\mathcal{G}-x}^{1}(w)$. Since $E \subset\left(\begin{array}{c}X \backslash\{x\} \\ r-1\end{array}\right)$ and $|E \cap B| \geq 1$, we conclude that

$$
\left|\operatorname{tr}_{\mathcal{G}-x}^{1}(w)\right| \leq|B|\left(\begin{array}{l}
n-2 \\
r-2
\end{array}\right)<3 \epsilon^{\prime \prime} n\left(\begin{array}{l}
n-2 \\
r-2
\end{array}\right)<3 \epsilon^{\prime \prime}\left(\begin{array}{l}
n-1 \\
r-1
\end{array}\right) .
$$

Claim 3. $\operatorname{tr}_{\mathcal{G}-x}^{0}(w)=\emptyset$.

Proof. Clearly $\operatorname{tr}_{\mathcal{G}-x}^{0}(w) \subset \mathcal{L}_{w} \cup \mathcal{S}_{w}$. We have already argued that $\mathcal{K}_{1}$ is a star with center $x$, thus $\operatorname{tr}_{\mathcal{G}-x}(w) \cap \mathcal{L}_{w}=\emptyset$. Now suppose that $E \in \operatorname{tr}_{\mathcal{G}-x}^{0}(w) \cap \mathcal{S}_{w}$. Then $E \cap B=\emptyset$, and hence by definition of $B$ we get $E \cap V\left(\mathcal{K}_{1}\right) \neq \emptyset$. Therefore $E \in \mathcal{K}_{1}^{\prime}$, where $\mathcal{K}_{1}^{\prime}$ is defined as in Lemma 3.1. By Lemma 3.1, $\mathcal{K}_{1}^{\prime}$ is intersecting, and thus 
every $F \in \mathcal{K}_{1}$ satisfies $F \cap E \neq \emptyset$. Since $F$ also contains $x$, and $x \notin E$, we obtain

$$
\left|\mathcal{K}_{1}\right|<|E|\left(\begin{array}{c}
n-2 \\
r-3
\end{array}\right)=(r-1)\left(\begin{array}{c}
n-2 \\
r-3
\end{array}\right),
$$

which contradicts (5.5). This completes the proof of the claim.

Claim 3, (5.10) and (5.9) imply that

$$
\left|\mathcal{G}_{1}\right| \leq\left|\operatorname{tr}_{\mathcal{G}-x}(w)\right|=\left|\operatorname{tr}_{\mathcal{G}-x}^{0}(w)\right|+\left|\operatorname{tr}_{\mathcal{G}-x}^{1}(w)\right|<3 \epsilon^{\prime \prime}\left(\begin{array}{c}
n-1 \\
r-1
\end{array}\right) .
$$

Now (5.11) and (5.8) together yield

$$
\begin{aligned}
|\mathcal{G}-x|=\left|\mathcal{G}_{1}\right|+\left|\mathcal{G}_{2}\right| & <3 \epsilon^{\prime \prime}\left(\begin{array}{c}
n-1 \\
r-1
\end{array}\right)+3 r \epsilon^{\prime \prime}\left(\begin{array}{c}
n \\
r-1
\end{array}\right) \\
& <r \epsilon^{\prime \prime}\left(\begin{array}{c}
n-1 \\
r-1
\end{array}\right)+4 r \epsilon^{\prime \prime}\left(\begin{array}{c}
n-1 \\
r-1
\end{array}\right)<\delta\left(\begin{array}{c}
n-1 \\
r-1
\end{array}\right),
\end{aligned}
$$

where the last inequality holds since $\epsilon^{\prime \prime} \leq \delta / 5 r$. Thus (5.7) holds, and the proof is complete in the case $r \geq 4$.

Step $2(r=3)$. Recall that $w$ was chosen to maximize $\left|\mathcal{L}_{w}\right|$, and therefore $\left|\mathcal{L}_{w}\right| \geq$ $(1-2 \epsilon)(n-2)$. By Lemma 3.1 each component of $\mathcal{L}_{w}$ is an intersecting family. Consequently, the graph spanned by the edges of a component is either $K_{2}$ (an edge), $K_{3}$ (a triangle), or $K_{1, p}$ for $p \geq 2$ (a nontrivial star). Let $\hat{\mathcal{K}}_{1}, \ldots, \hat{\mathcal{K}}_{\hat{s}}$ be the components isomorphic to $K_{2}$, let $\tilde{\mathcal{K}}_{1}, \ldots, \tilde{\mathcal{K}}_{\tilde{s}}$ be the components isomorphic to $K_{3}$, and let $\mathcal{K}_{1}, \ldots, \mathcal{K}_{s}$ be the components isomorphic to some nontrivial star. Set $n_{i}=n\left(\mathcal{K}_{i}\right)$ and assume that $n_{1} \geq n_{2} \geq \cdots \geq n_{s}$.

Our approach is to prove that $n_{1} \geq\left(1-\epsilon^{\prime}\right) n$. Let

$$
B_{1}=X \backslash\left(V\left(\mathcal{L}_{w}\right) \cup\{w\}\right) .
$$

Since the number of edges in each component of $\mathcal{L}_{w}$ is at most its number of vertices, (5.1) implies that $n\left(\mathcal{L}_{w}\right) \geq\left|\mathcal{L}_{w}\right| \geq(1-2 \epsilon)(n-2)$, and we obtain

$$
\left|B_{1}\right|<3 \epsilon n \text {. }
$$

Partition $\mathcal{G}-w$ into $\mathcal{G}_{0} \cup \mathcal{G}_{1} \cup \mathcal{G}_{2} \cup \mathcal{G}_{3}$, where

$$
\mathcal{G}_{i}=\left\{E \in \mathcal{G}-w:\left|E \cap B_{1}\right|=i\right\} \quad \text { for each } i .
$$

By Proposition 3.3 .

$$
\left|\mathcal{G}_{2} \cup \mathcal{G}_{3}\right|<9 \epsilon\left(\begin{array}{l}
n \\
2
\end{array}\right) .
$$

We now focus on $\mathcal{G}_{0} \cup \mathcal{G}_{1}$. In what follows we use the notation " $G$-component" to mean a component isomorphic to $G$.

Claim 4. Every $E \in \mathcal{G}_{0} \cup \mathcal{G}_{1}$ has a nonempty intersection with at most one component of $\mathcal{L}_{w}$. Moreover, if $|E \cap V(\mathcal{K})| \geq 2$ for some component $\mathcal{K}$ of $\mathcal{L}_{w}$, then either

1) $\mathcal{K}=\mathcal{K}_{i}$ for some $i$, and $x_{i} \in E$, where $x_{i}$ is the center of $\mathcal{K}_{i}$, or

2) $E=V(\mathcal{K})$, where $\mathcal{K}$ is a $K_{3}$-component, or

3) $E \backslash B_{1}=V(\mathcal{K})$, where $\mathcal{K}$ is a $K_{2}$-component. 
Proof. Suppose, for a contradiction, that both $E \cap V(\mathcal{K})$ and $E \cap V(\mathcal{H})$ are nonempty for some two components $\mathcal{K}, \mathcal{H}$ of $\mathcal{L}_{w}$. Then there exist (2-element) sets $E_{\mathcal{K}} \in \mathcal{K}$ and $E_{\mathcal{H}} \in \mathcal{H}$ such that both $E \cap E_{\mathcal{K}}$ and $E \cap E_{\mathcal{H}}$ are nonempty. Since $w \notin E$ and $E_{\mathcal{K}} \cap E_{\mathcal{H}}=\emptyset$, the sets

$$
E_{\mathcal{K}} \cup\{w\}, E_{\mathcal{H}} \cup\{w\}, E
$$

from a triangle in $\mathcal{G}$.

For 1) assume that there are two leaves $l, l^{\prime} \in E \cap \mathcal{K}_{i}$, and $x_{i} \notin E$. Then the sets

$$
\left\{w, x_{i}, l\right\},\left\{w, x_{i}, l^{\prime}\right\}, E
$$

form a triangle in $\mathcal{G}$, a contradiction. Therefore $x_{i} \in E$ as claimed. For 2), suppose that $\left|E \cap \tilde{\mathcal{K}}_{i}\right|=2$ for some $K_{3}$-component $\tilde{\mathcal{K}}_{i}$. Let $\{a, b\} \in V\left(\tilde{\mathcal{K}}_{i}\right) \cap E$, and let $c$ be the third element of $V\left(\tilde{\mathcal{K}}_{i}\right)$. Then

$$
\{w, c, a\},\{w, c, b\}, E
$$

is a triangle in $\mathcal{G}$, a contradiction. Finally, if $E \supset V(\mathcal{K})$ for some $K_{2}$-component $\mathcal{K}$, then the first part of the claim implies that the third vertex of $E$ is in $B_{1}$. Thus $E \backslash B=V(\mathcal{K})$.

Claim 4 allows us to estimate the size of $\mathcal{G}_{1}$.

Claim 5. $\left|\mathcal{G}_{1}\right|<7 \epsilon\left(\begin{array}{l}n \\ 2\end{array}\right)$.

Proof. Let $E \in \mathcal{G}_{1}$. Then $\left|E \cap V\left(\mathcal{L}_{w}\right)\right|=2$, and Claim 4 implies that $E \cap V\left(\mathcal{L}_{w}\right)$ lies within a component $\mathcal{K}$ of $\mathcal{L}_{w}$. The second part of Claim 4 implies that $E \cap V\left(\mathcal{L}_{w}\right)$ is a set of some component of $\mathcal{L}_{w}$. This yields

$$
\left|\mathcal{G}_{1}\right|<\left|\mathcal{L}_{w}\right|\left|B_{1}\right|<n(3 \epsilon n)<7 \epsilon\left(\begin{array}{l}
n \\
2
\end{array}\right),
$$

since $n>7$, completing the proof of the claim.

Now we turn to $\mathcal{L}_{w} \cup \mathcal{S}_{w} \cup \mathcal{G}_{0}$. Partition $\mathcal{S}_{w}$ into $\mathcal{S}_{w}^{0} \cup \mathcal{S}_{w}^{1} \cup \mathcal{S}_{w}^{2}$, where

$$
\mathcal{S}_{w}^{i}=\left\{E \in \mathcal{S}_{w}:\left|E \cap B_{1}\right|=i\right\}
$$

Clearly

$$
\left|\mathcal{S}_{w}^{1} \cup \mathcal{S}_{w}^{2}\right| \leq\left|B_{1}\right| n<3 \epsilon n^{2} .
$$

A set in $\mathcal{G}$ either contains or omits $w$. Therefore (5.13), Claim 5 and $n>n_{0}$ give

$$
\begin{aligned}
|\mathcal{G}| & =\left|\mathcal{G}_{2} \cup \mathcal{G}_{3}\right|+\left|\mathcal{G}_{1}\right|+\left|\mathcal{G}_{0}\right|+\left|\mathcal{L}_{w}\right|+\left|\mathcal{S}_{w}\right| \\
& <9 \epsilon\left(\begin{array}{l}
n \\
2
\end{array}\right)+7 \epsilon\left(\begin{array}{l}
n \\
2
\end{array}\right)+\left|\mathcal{G}_{0}\right|+n+\left|\mathcal{S}_{w}\right| \\
& <17 \epsilon\left(\begin{array}{l}
n \\
2
\end{array}\right)+\left|\mathcal{G}_{0}\right|+\left|\mathcal{S}_{w}\right| .
\end{aligned}
$$

Since $|\mathcal{G}|>(1-\epsilon)\left(\begin{array}{c}n-1 \\ 2\end{array}\right)$, we conclude from $n>n_{0}$ that

$$
\left|\mathcal{G}_{0}\right|+\left|\mathcal{S}_{w}\right|>(1-\epsilon)\left(\begin{array}{c}
n-1 \\
2
\end{array}\right)-17 \epsilon\left(\begin{array}{l}
n \\
2
\end{array}\right)>(1-19 \epsilon)\left(\begin{array}{l}
n \\
2
\end{array}\right) .
$$

Given $E \in \mathcal{G}_{0}$, Claim 4 implies that either $E \subset V\left(\mathcal{K}_{i}\right)$ for some $i$, and $x_{i} \in E$, where $x_{i}$ is the center of the star $\mathcal{K}_{i}$, or $E=V(\mathcal{K})$ for some $K_{3}$-component $\mathcal{K}$. If $E=\left\{x_{i}, l_{i}, l_{i}^{\prime}\right\} \subset V\left(\mathcal{K}_{i}\right)$ for some nontrivial star component, we may associate the pair $\left\{l_{i}, l_{i}^{\prime}\right\}$ to $E$, and this mapping is injective (since a nontrivial star has only one center). Let $\partial^{\prime} \mathcal{G}_{0}$ be the set of all such pairs $\left\{l_{i}, l_{i}^{\prime}\right\}$. 
Claim 6. $\partial^{\prime} \mathcal{G}_{0} \cap \mathcal{S}_{w}^{0}=\emptyset$.

Proof. Suppose to the contrary that $\left\{l_{i}, l_{i}^{\prime}\right\} \in \partial^{\prime} \mathcal{G}_{0} \cap \mathcal{S}_{w}^{0}$. Then $\left\{l_{i}, l_{i}^{\prime}, x_{i}\right\}$ and $\left\{l_{i}, l_{i}^{\prime}, w\right\}$ are both sets in $\mathcal{G}$. Therefore $\left\{l_{i}, l_{i}^{\prime}\right\} \in \mathcal{L}_{w}$, since two sets of $\mathcal{G}$ contain this pair. Hence $\left\{l_{i}, l_{i}^{\prime}\right\} \notin \mathcal{S}_{w}$ and $\left\{l_{i}, l_{i}^{\prime}\right\} \notin \mathcal{S}_{w}^{0}$, a contradiction.

Let $\overline{\mathcal{S}_{w}^{0}}=\left({ }_{2}\left(\mathcal{L}_{w}\right)\right) \backslash \mathcal{S}_{w}^{0}$ and $\overline{\partial^{\prime} \mathcal{G}_{0}}=\left({ }^{V}\left(\mathcal{L}_{w}\right)\right) \backslash \partial^{\prime} \mathcal{G}_{0}$. Then Claim 6, (5.14), and the discussion before Claim 6 imply that

$$
\begin{gathered}
\left|\mathcal{G}_{0}\right|+\left|\mathcal{S}_{w}\right|=\left|\partial^{\prime} \mathcal{G}_{0}\right|+\tilde{s}+\left|\mathcal{S}_{w}^{0}\right|+\left|\mathcal{S}_{w}^{1} \cup \mathcal{S}_{w}^{2}\right|<\left|\partial^{\prime} \mathcal{G}_{0}\right|+\tilde{s}+\left|\mathcal{S}_{w}^{0}\right|+3 \epsilon n^{2} \\
<\left(\begin{array}{c}
n \\
2
\end{array}\right)-\left|\overline{\mathcal{S}_{w}^{0}} \cap \overline{\partial^{\prime} \mathcal{G}_{0}}\right|+3 \epsilon n^{2}+n .
\end{gathered}
$$

Together with (5.15) and $n>n_{0}$, this implies that

$$
\left|\overline{\mathcal{S}_{w}^{0}} \cap \overline{\partial^{\prime} \mathcal{G}_{0}}\right|<\left(\begin{array}{l}
n \\
2
\end{array}\right)-(1-19 \epsilon)\left(\begin{array}{l}
n \\
2
\end{array}\right)+3 \epsilon n^{2}+n<26 \epsilon\left(\begin{array}{l}
n \\
2
\end{array}\right) .
$$

Claim 7. $\hat{s}+3 \tilde{s}<10 \sqrt{\epsilon}$.

Proof. We will show that the number of vertices in $K_{2}$ - and $K_{3}$-components, namely $\hat{s}+3 \tilde{s}$, is small. We will also show that if $\hat{s}+3 \tilde{s} \geq 10 \sqrt{\epsilon} n$, then $\left|\overline{\mathcal{S}_{w}^{0}} \cap \overline{\partial^{\prime} \mathcal{G}_{0}}\right|>26 \epsilon\left(\begin{array}{l}n \\ 2\end{array}\right)$, contradicting (5.16). Consider a $K_{2}$-component $\hat{\mathcal{K}}_{i}=\{\{a, b\}\}$, and suppose that $a \in E_{a} \in \mathcal{S}_{w}^{0}$ and $b \in E_{b} \in \mathcal{S}_{w}^{0}$ for some $E_{a}, E_{b}$. If $E_{a} \cap E_{b}=\emptyset$, then the argument in the proof of Lemma 3.1 yields a triangle in $\mathcal{G}$. If $E_{a} \cap E_{b}=\{c\}$, then the same argument applies unless $\{a, b, c\} \in \mathcal{G}$. But then $\{a, c\}$ is contained in two edges of $\mathcal{G}(\{a, b, c\}$ and $\{w, a, c\})$, which contradicts the fact that $\{a, c\}=E_{a} \in \mathcal{S}_{w}^{0} \subset \mathcal{S}_{w}$.

We may therefore assume that at least one vertex in each $K_{2}$-component $\hat{\mathcal{K}}_{i}$ lies in no set of $\mathcal{S}_{w}^{0}$. By definition of $\partial^{\prime} \mathcal{G}_{0}$, no vertex of a component of $\hat{\mathcal{K}}_{i}$ lies in a pair from $\partial^{\prime} \mathcal{G}_{0}$. We may therefore pick one element from each $\hat{\mathcal{K}}_{i}$ so that the resulting set $\hat{S}$ of size $\hat{s}$ satisfies the following two conditions:

1) $\overline{\mathcal{S}_{w}^{0}} \cap \overline{\partial^{\prime} \mathcal{G}_{0}}$ contains all edges (2-element sets) with both endpoints in $\hat{S}$, and

2) $\frac{w}{\mathcal{S}_{w}^{0}} \cap \overline{\partial^{\prime} \mathcal{G}_{0}}$ contains all edges with one endpoint in $\hat{S}$ and the other endpoint in $\bigcup_{i=1}^{\hat{s}} V\left(\hat{\mathcal{K}}_{i}\right) \backslash \hat{S}$.

This gives $\left(\begin{array}{c}\hat{s} \\ 2\end{array}\right)+\hat{s}(\hat{s}-1)$ members of $\overline{\mathcal{S}_{w}^{0}} \cap \overline{\partial^{\prime} \mathcal{G}_{0}}$. We next exhibit another $\left(\begin{array}{c}3 \tilde{s} \\ 2\end{array}\right)+6 \hat{s} \tilde{s}$ members of $\overline{\mathcal{S}_{w}^{0}} \cap \overline{\partial^{\prime} \mathcal{G}_{0}}$.

Suppose that $E$ is a 2-element set contained in the union of the vertex sets of the $K_{2^{-}}$and $K_{3}$-components of $\mathcal{L}_{w}$, with at least one element in some $K_{3}$-component. Then Lemma 3.1 and the definition of $\partial^{\prime} \mathcal{G}_{0}$ imply that $E \subset \overline{\mathcal{S}_{w}^{0}} \cap \overline{\partial^{\prime} \mathcal{G}_{0}}$. Putting these observations together and using $n>n_{0}$ yields

$$
\left|\overline{\mathcal{S}_{w}^{0}} \cap \overline{\partial^{\prime} \mathcal{G}_{0}}\right| \geq\left(\begin{array}{c}
\hat{s} \\
2
\end{array}\right)+\hat{s}(\hat{s}-1)+\left(\begin{array}{c}
3 \tilde{s} \\
2
\end{array}\right)+6 \hat{s} \tilde{s}>\left(\frac{\hat{s}+3 \tilde{s}}{2}\right)^{2}>(5 \sqrt{\epsilon} n)^{2}>26 \epsilon\left(\begin{array}{l}
n \\
2
\end{array}\right) .
$$

This is the contradiction we desired.

Let

$$
B_{2}=\bigcup_{i=1}^{\hat{s}} V\left(\hat{\mathcal{K}}_{i}\right) \cup \bigcup_{i=1}^{\tilde{s}} V\left(\tilde{\mathcal{K}}_{i}\right) \cup B_{1} .
$$

Thus

$$
X \backslash B_{2}=\bigcup_{i=1}^{s} V\left(\mathcal{K}_{i}\right) \cup\{w\}
$$


Then Claim 7, (5.12) and $n>n_{0}$ imply that

$$
\left|B_{2}\right|=\hat{s}+3 \tilde{s}+\left|B_{1}\right|<10 \sqrt{\epsilon} n+3 \epsilon n<11 \sqrt{\epsilon} n .
$$

Partition $\mathcal{G}$ into $\mathcal{G}_{B_{2}}^{1} \cup \mathcal{G}_{B_{2}}^{2}$, where

$$
\mathcal{G}_{B_{2}}^{1}=\left\{E \in \mathcal{G}:\left|E \cap B_{2}\right| \leq 1\right\} \quad \text { and } \quad \mathcal{G}_{B_{2}}^{2}=\left\{E \in \mathcal{G}:\left|E \cap B_{2}\right| \geq 2\right\} .
$$

Proposition 3.3 and (5.17) imply that

$$
\left|\mathcal{G}_{B_{2}}^{2}\right| \leq 33 \sqrt{\epsilon}\left(\begin{array}{l}
n \\
2
\end{array}\right) .
$$

Therefore, since $n>n_{0}$,

$$
\left|\mathcal{G}_{B_{2}}^{1}\right|=|\mathcal{G}|-\left|\mathcal{G}_{B_{2}}^{2}\right| \geq(1-\epsilon)\left(\begin{array}{c}
n-1 \\
2
\end{array}\right)-33 \sqrt{\epsilon}\left(\begin{array}{l}
n \\
2
\end{array}\right)>(1-34 \sqrt{\epsilon})\left(\begin{array}{c}
n-1 \\
2
\end{array}\right)
$$

Since $B_{1} \subset B_{2}$, it follows that $\mathcal{G}_{B_{2}}^{1} \subset \mathcal{G}_{0} \cup \mathcal{G}_{1}$. Therefore, if $E \in \mathcal{G}_{B_{2}}^{1}$, we may apply Claim 4 to $E$. Either $\left|E \cap B_{2}\right|=1$, and the remaining two points of $E$ form a set in a nontrivial star component of $\mathcal{L}_{w}$, or $\left|E \cap B_{2}\right|=0$ and $x_{i} \in E \subset V\left(\mathcal{K}_{i}\right)$, where $x_{i}$ is the center of some $\mathcal{K}_{i}$. Consequently,

$$
\left|\mathcal{G}_{B_{2}}^{1}\right| \leq\left|B_{2}\right| \sum_{i=1}^{s}\left(n_{i}-1\right)+\sum_{i=1}^{s}\left(\begin{array}{c}
n_{i}-1 \\
2
\end{array}\right)<23 \sqrt{\epsilon}\left(\begin{array}{c}
n-1 \\
2
\end{array}\right)+\sum_{i=1}^{s}\left(\begin{array}{c}
n_{i}-1 \\
2
\end{array}\right),
$$

where the last inequality is implied by (5.17) and $n>n_{0}$. We thus obtain from (15.18) that

$$
\sum_{i=1}^{s}\left(\begin{array}{c}
n_{i} \\
2
\end{array}\right)>\sum_{i=1}^{s}\left(\begin{array}{c}
n_{i}-1 \\
2
\end{array}\right)>\left|\mathcal{G}_{B_{2}}^{1}\right|-23 \sqrt{\epsilon}\left(\begin{array}{c}
n-1 \\
2
\end{array}\right)>(1-57 \sqrt{\epsilon})\left(\begin{array}{c}
n-1 \\
2
\end{array}\right) .
$$

Lemma 4.2 with $t=4$ now implies that

$$
n_{1}>\left(1-\epsilon^{\prime}\right)(n-1)
$$

since $\epsilon \leq\left(\epsilon^{\prime} / 114\right)^{2}$. Let $x$ be the center of $\mathcal{K}_{1}$. This concludes Step 2 .

Step $3(r=3)$. We are now in a position to finish the proof. We will show that $|\mathcal{G}-x|<\delta\left(\begin{array}{c}n-1 \\ 2\end{array}\right)$. Set

$$
B=B_{2} \cup\{w\}=X \backslash V\left(\mathcal{K}_{1}\right) .
$$

Then (5.19) and $n>n_{0}$ imply that

$$
|B| \leq 2 \epsilon^{\prime} n .
$$

Partition $\mathcal{G}-x$ into $\mathcal{G}_{B}^{1} \cup \mathcal{G}_{B}^{2}$, where

$$
\mathcal{G}_{B}^{1}=\{E \in \mathcal{G}-x:|E \cap B| \leq 1\} \quad \text { and } \quad \mathcal{G}_{B}^{2}=\{E \in \mathcal{G}-x:|E \cap B| \geq 2\} .
$$

Proposition 3.3, (5.20) and $n>n_{0}$ imply that

$$
\left|\mathcal{G}_{B}^{2}\right| \leq 6 \epsilon^{\prime}\left(\begin{array}{l}
n \\
2
\end{array}\right) .
$$

On the other hand,

Claim 8. $\mathcal{G}_{B}^{1}=\emptyset$. 
Proof. Suppose that $E \in \mathcal{G}_{B}^{1}$. If $w \notin E$, then since $\mathcal{G}_{B}^{1} \subset \mathcal{G}_{0} \cup \mathcal{G}_{1}$, Claim 4, part 1) implies that $x \in E$, which contradicts the definition of $\mathcal{G}_{B}^{1}$. On the other hand, if $w \in E$, then $E \backslash\{w\} \in \mathcal{L}_{w} \cup \mathcal{S}_{w}$, and in particular, $E \backslash\{w\} \in \mathcal{K}_{1}^{\prime}$. Lemma 3.1 now implies that $\mathcal{K}_{1} \cup(E \backslash\{w\})$ is an intersecting family. Since $n_{1} \geq 4$, this implies that $E \backslash\{w\} \in \mathcal{K}_{1}$ (i.e., $E \backslash\{w\}$ is a 2-element set of $\mathcal{K}_{1}$ ), and in particular, that $x \in E$. This again contradicts the definition of $\mathcal{G}_{B}^{1}$, and completes the proof of the claim.

From (5.21) and Claim 8 we obtain

$$
|\mathcal{G}-x| \leq\left|\mathcal{G}_{B}^{1}\right|+\left|\mathcal{G}_{B}^{2}\right| \leq 6 \epsilon^{\prime}\left(\begin{array}{l}
n \\
2
\end{array}\right) \leq \delta\left(\begin{array}{l}
n \\
2
\end{array}\right) .
$$

If $S=X \backslash\{x\}$, then $|S|=n-1$, and the number of edges of $\mathcal{G}$ contained in $S$ is at most $\delta\left(\begin{array}{c}n-1 \\ 2\end{array}\right)$, as required by the theorem.

\section{Concluding REMARKS AND OPEN PROBlems}

As described in the Introduction, studying the stability of monotone properties, apart from shedding more light on the extremal problem being investigated, sometimes leads to unexpected consequences. It also provides a common framework for several classical results. Nevertheless, the theory is in its incipient stages. A noteworthy aspect that makes these questions more interesting is that it is likely that many classical extremal problems are not $t$-stable for any fixed $t$. We present two examples.

Example 1. The most basic of all results in extremal set theory is that if $\mathcal{G} \subset 2^{[n]}$ is an intersecting family, then $|\mathcal{G}| \leq 2^{n-1}$. It is well known that there are many extremal examples, since every intersecting family of size less than $2^{n-1}$ can be augmented to one of size $2^{n-1}$. Nevertheless, this does not a priori prohibit this property from being $t$-stable for some $t$. In particular, there are two canonical extremal examples for this problem when $n$ is odd: $\mathcal{H}_{1}=\bigcup_{i>n / 2}\left(\begin{array}{c}{[n]} \\ i\end{array}\right)$ and $\mathcal{H}_{2}$ which is a star of size $2^{n-1}$. One could ask whether this property is 2 -stable, with respect to these two structures. However, the following example shows this to be false. Let $\mathcal{G}_{1}$ consist of all sets of the form $S \cup\{n\}$, where $S \subset[n-1]$ with $n / 2-\sqrt{n}<|S|<n / 2-2$. Let $\mathcal{G}_{2}$ consist of all sets of size at least $n / 2+\sqrt{n}+2$. Then it is clear that $\mathcal{G}=\mathcal{G}_{1} \cup \mathcal{G}_{2}$ is an intersecting family, and it can be augmented to one of size $2^{n-1}$. But $\mathcal{G}$ also shows that this property is not stable with respect to $\mathcal{H}_{1}$ and $\mathcal{H}_{2}$. One can modify this construction and in fact argue that this property is not $t$-stable for any $t$.

Example 2. Sperner's theorem, stating that every maximum antichain in $2^{[n]}$ is either $\left(\begin{array}{c}{[n]} \\ \lfloor n / 2\rfloor\end{array}\right)$ or $\left(\begin{array}{c}{[n]} \\ {[n / 2\rceil}\end{array}\right)$, does not seem to have a stability version. Indeed (as pointed out by G. Turán), one can take the family $\mathcal{G}=\left(\begin{array}{c}{[n]} \\ \lfloor n / 2\rfloor-1\end{array}\right)$, which is almost the same size as $\left(\begin{array}{c}{[n]} \\ \lfloor n / 2\rfloor\end{array}\right)$, but cannot be transformed to $\left(\begin{array}{c}{[n]} \\ \lfloor n / 2\rfloor\end{array}\right)$ by adding or deleting a small proportion of its sets. This argument can be extended to show that this property is not stable and in fact not $t$-stable for any $t>1$.

In light of the two examples above, it would be very interesting to find a nontrivial problem in extremal set theory dealing with nonuniform systems that is $t$-stable for some $t$. Perhaps one candidate is the nonuniform analogue of the problem considered in this paper. A result of Erdős and Milner (see Lossers [15]) states that if $\mathcal{G} \subset 2^{[n]} \backslash \emptyset$ is triangle-free (meaning $\mathcal{G}$ contains no three sets $A, B, C$ with pairwise 
nonempty intersection but empty total intersection), then $|\mathcal{G}| \leq 2^{n-1}+n-1$. Recently, the author and Verstraëte [18] proved that the unique extremal example is a star of size $2^{n-1}$ together with the remaining $n-1$-element sets. Perhaps all near extremal structures have a similar structure, and a stability version holds.

Problem. Determine if the property of being triangle-free (for nonuniform systems) is stable or $t$-stable for some $t>1$.

It seems that problems about uniform families tend more readily to have stability analogues. We have already mentioned the Erdős-Simonovits stability theorem for graphs and the Hilton-Milner theorem which implies (in a strong form) that intersecting $r$-uniform set systems are stable. In fact, the Hilton-Milner theorem itself has a stability version.

Example 3. A nontrivial intersecting family is an intersecting family $\mathcal{F}$ with $\tau(\mathcal{F})>1$. Define $\mathcal{G}_{1}=\left\{G \in\left(\begin{array}{c}{[n]} \\ r\end{array}\right): 1 \in G, G \cap[2, r+1] \neq \emptyset\right\} \cup\{[2, r+1]\}$, and $\mathcal{G}_{2}=\left\{G \in\left(\begin{array}{c}{[n]} \\ r\end{array}\right):|[3] \cap G| \geq 2\right\}$. For $r=2, \mathcal{G}_{1} \equiv \mathcal{G}_{2}$, for $r=3,\left|\mathcal{G}_{1}\right|=\left|\mathcal{G}_{2}\right|$, and for $r \geq 4, n>2 r$, we have $\left|\mathcal{G}_{1}\right|>\left|\mathcal{G}_{2}\right|$. The Hilton-Milner theorem states that if $n>2 r$ and $\mathcal{G} \subset\left(\begin{array}{c}{[n]} \\ r\end{array}\right)$ is a nontrivial intersecting family, then

$$
|\mathcal{G}| \leq\left|\mathcal{G}_{1}\right|=\left(\begin{array}{c}
n-1 \\
r-1
\end{array}\right)-\left(\begin{array}{c}
n-r-1 \\
r-1
\end{array}\right)+1 .
$$

Moreover, equality holds only for $\mathcal{G}_{1}$ or $\mathcal{G}_{2}$. Although the property of being a nontrivial intersecting family is not monotone, we can still talk about stability. A recent result of Sanders (Lemma 7 in [20]) implies the following result.

Theorem 6.1. The property $\mathcal{P}^{r}$ of being a nontrivial intersecting family is 2-stable for $r=3$ and stable for $r \geq 4$.

Example 4. Erdős defined $f_{r}(n)$ to be the maximum size of a family of $r$-sets on an $n$ element set containing no two pairs of disjoint $r$-sets with the same union. Answering a question of Erdös, Füredi [10] proved that $f_{r}(n) \leq \frac{7}{2}\left(\begin{array}{c}n \\ r-1\end{array}\right)$. The author and Verstraëte [17] slightly improved Füredi's result by showing that $f_{r}(n)<$ $3\left(\begin{array}{c}n \\ r-1\end{array}\right)$. Füredi further conjectured that $f_{3}(n)=\left(\begin{array}{l}n \\ 2\end{array}\right)$ for infinitely many $n$ and for $r \geq 4$,

$$
f_{r}(n)=\left(\begin{array}{c}
n-1 \\
r-1
\end{array}\right)+\left\lfloor\frac{n-1}{r}\right\rfloor
$$

for all sufficiently large $n$. His conjecture was motivated by the lower bound obtained from a star plus a matching for $r \geq 4$, and a set system obtained from a 5-design for $r=3$. For $r=3$ the situation seems complicated, but perhaps for $r=4$, the star is essentially the only example.

Conjecture 6.2. For $r \geq 4$, the property $\mathcal{P}^{r}$ of containing no two pairs of disjoint sets with the same union is stable.

\section{ACKNOWLEDGMENTS}

Some of the ideas in Step $2(r=3)$ were obtained during discussions with J. Verstraëte while preparing the paper [18]. I am grateful for those conversations. Thanks also to A. Gyárfás for helpful discussions regarding Example 1 in Section 6, G. Turán for the same regarding Example 2, and a referee for many helpful comments. 


\section{REFERENCES}

[1] J. Balogh, B. Bollobás, M. Simonovits, The number of graphs without forbidden subgraphs, Journal of Combinatorial Theory, Series B., 91 (2004), no. 1, 1-24. MR2047528 (2005b:05122)

[2] J.-C. Bermond, P. Frankl, On a conjecture of Chvátal on $m$-intersecting hypergraphs. Bull. London Math. Soc. 9 (1977), no. 3, 310-312. MR0457287(56:15495)

[3] V. Chvátal, An extremal set-intersection theorem, J. London Math. Soc. (2) 9 (1974/75), 355-359. MR0354388 (50:6867)

[4] P. Erdős, Topics in combinatorial analysis. Proc. Second Louisiana Conf. on Comb., Graph Theory and Computing (R. C. Mullin et al., eds.) pp. 2-20, Louisiana State Univ., Baton Rouge, 1971.

[5] P. Erdős, H. Ko, R. Rado, Intersection theorems for systems of finite sets. Quart. J. Math. Oxford Ser. (2) 12 (1961), 313-320. MR0140419(25:3839)

[6] P. Erdős, D. J. Kleitman, B. L. Rothschild, Asymptotic enumeration of $K_{n}$-free graphs. (English. Italian summary) Colloquio Internazionale sulle Teorie Combinatorie (Rome, 1973), Tomo II, pp. 19-27. Atti dei Convegni Lincei, No. 17, Accad. Naz. Lincei, Rome, 1976. MR0463020 (57:2984)

[7] P. Frankl, On Sperner families satisfying an additional condition, J. Combin. Theory Ser. A, 20 (1976), no. 1, 1-11. MR0398842 (53:2693)

[8] P. Frankl, On a problem of Chvátal and Erdős on hypergraphs containing no generalized simplex. J. Combin. Theory Ser. A 30 (1981), no. 2, 169-182. MR0611249 (83k:05086)

[9] P. Frankl, Z. Füredi, Exact solution of some Turán-type problems, J. Combin. Theory Ser. A, 45 (1987), no. 2, 226-262. MR0894820 (88h:05005)

[10] Z. Füredi, Hypergraphs in which all disjoint pairs have distinct unions. Combinatorica, 4 (1984), no. 2-3, 161-168. MR0771723 (86c:05009)

[11] Z. Füredi, M. Simonovits, Triple systems not containing a Fano configuration, Combin. Probab. Comput. 14 (2005), no. 4, 467-484. MR2160414(2006d:05034)

[12] A. J. W. Hilton, E. C. Milner, Some intersection theorems for systems of finite sets. Quart. J. Math. Oxford Ser. (2) 18 (1967), 369-384. MR0219428 (36:2510)

[13] P. Keevash, B. Sudakov, On a hypergraph Turán problem of Frankl, Combinatorica, 25 (2005), no. 6, 673-706. MR2199431

[14] P. Keevash, B. Sudakov, The Turán number of the Fano plane, Combinatorica, 25 (2005), no. 5, 561-574. MR2176425 (2006f:05093)

[15] O. P. Lossers, P. Erdos, E. Milner, Canad. Math Bull. 16 (1973), 145-146.

[16] D. Mubayi, O. Pikhurko, A new generalization of Mantel's theorem to $k$-graphs, submitted.

[17] D. Mubayi, J. Verstraëte, A hypergraph extension of the bipartite Turán problem, J. Combin. Theory Ser. A, 106 (2004), no. 2, 237-253. MR2021684(2005b:05128)

[18] D. Mubayi, J. Verstraëte, Proof of a conjecture of Erdős on triangles in set-systems, Combinatorica, 25 (2005), no. 5, 599-614. MR2176427

[19] O. Pikhurko, Exact computation of the hypergraph Turan function for expanded complete 2-graphs, 9 pp., accepted J. Combin Theory Ser. B, publication suspended for an indefinite time; see http://www.math.cmu.edu/ pikhurko/Copyright.html

[20] A. J. Sanders, Covering by intersecting families, J. Combin. Theory Ser. A 108 (2004), no. 1, 51-61. MR2087304 (2005d:05045)

[21] M. Simonovits, A method for solving extremal problems in graph theory, stability problems. 1968 Theory of Graphs (Proc. Colloq., Tihany, 1966) pp. 279-319, Academic Press, New York. MR 0233735 (38:2056)

Department of Mathematics, Statistics, \& Computer Science, University of Illinois at Chicago, 851 S. Morgan Street, Chicago, Illinois 60607-7045

E-mail address: mubayi@math.uic.edu 